Acta Crystallographica Section E

Structure Reports

Online
ISSN 1600-5368

\section{1-(4-Nitrobenzoyl)thiosemicarbazide monohydrate: a three-dimensional hydrogen-bonded framework structure}

In the title compound, $\mathrm{C}_{8} \mathrm{H}_{8} \mathrm{~N}_{4} \mathrm{O}_{3} \mathrm{~S} \cdot \mathrm{H}_{2} \mathrm{O}$, strong hydrogen bonding results in the formation of a number of chains and Núbia Boechat, ${ }^{a}$ Adriana Lages, ${ }^{a}$ W. Bruce Kover, Solange M. S. V. Wardell ${ }^{\mathrm{a}}$ and Janet M. S. Skakle ${ }^{\text {C* }}$

${ }^{\text {a}}$ Fundação Oswaldo Cruz, Instituto de Tecnologia em Fármacos, Departamento de Síntese Orgânica, Manguinhos, CEP 21041-250 Rio de Janeiro, RJ, Brazil, 'b Departamento de Química Oorgânica, Instituto de Química, Universidade Federal do Rio de Janeiro, 21945-970 Rio de Janeiro, RJ, Brazil, and cDepartment of Chemistry, College of Physical Sciences, University of Aberdeen, Meston Walk, Aberdeen AB24 3UE, Scotland

Correspondence e-mail: j.skakle@abdn.ac.uk

\section{Key indicators}

Single-crystal X-ray study

$T=120 \mathrm{~K}$

Mean $\sigma(\mathrm{C}-\mathrm{C})=0.002 \AA$

$R$ factor $=0.031$

$w R$ factor $=0.091$

Data-to-parameter ratio $=14.1$

For details of how these key indicators were automatically derived from the article, see http://journals.iucr.org/e.
Accepted 23 May 2006
(C) 2006 International Union of Crystallography All rights reserved dimers, which combine to give a three-dimensional hydrogenbonded framework.

\section{Comment}

Acylthiosemicarbazides are versatile compounds, having a large spectrum of biological properties (Bhat et al., 1967; Guersoy \& Karali, 1995; Plumitallo et al., 2004). They are, in addition, useful precursors of various biologically active heterocyclic compounds, including triazoles (Kane et al., 1994; Palaska et al., 2002), thiadiazoles (Oruc et al., 2004; Palaska et al., 2002) and oxadiazoles (Palaska et al., 2002; Yale \& Losee, 1966). Certain acylthiosemicarbazide-transition metal complexes have also been shown to possess useful biological activities (Shen et al., 1997; Singh \& Singh, 2001). As part of our interest in acylthiosemicarbazide compounds, we now report the crystal structure of 1-(4-nitrobenzoyl)thiosemicarbazide monohydrate, (I).

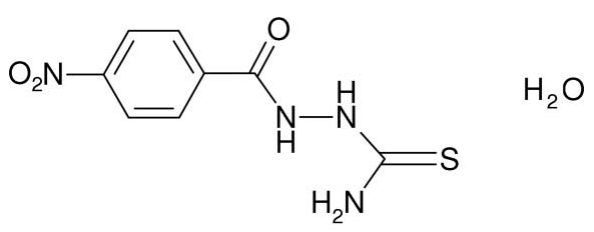

(I)

Within the asymmetric unit of (I), the $\mathrm{O}$ atom of the solvent water molecule acts as an $\mathrm{H}$-atom acceptor for the amide group of the organic molecule (Fig. 1). The $p$-nitro group is rotated from the essentially planar aryl group by an angle of $13.07(12)^{\circ}$, whereas the $\mathrm{CN}(\mathrm{O})$ group is twisted by $10.77(12)^{\circ}$.

The hydrogen bonding (Table 2) at the basic level produces a mixture of chains and dimers. The combination of the hydrogen bond described above, together with $\mathrm{O} 1 W-$ $\mathrm{H} 1 W A \cdots \mathrm{O} 7^{\mathrm{ii}}$ [symmetry code: (ii) $\left.x+1, y, z\right]$ leads to a $C_{2}^{2}(9)$ chain (Bernstein et al., 1995) along [010]. Another chain, $C(12)$, forms along [100] via the $\mathrm{N} 9-\mathrm{H} 9 A \cdots \mathrm{O} 42^{\mathrm{v}}$ hydrogen bond [symmetry code: (v) $x, y, z-1$ ]. These combine to form an $R_{5}^{6}(34)$ ring (Fig. 2); the disparity between the number of donors and acceptors results from the amide acting as a double donor. The rings link to create a sheet normal to [010] (Fig. 2).

All other hydrogen bonds involve $\mathrm{S}$ as an acceptor and result in dimers. In the first, the hydrogen bond within the asymmetric unit combines with $\mathrm{O} 1 W-\mathrm{H} 1 W A \cdots \mathrm{S} 1^{1}$ 


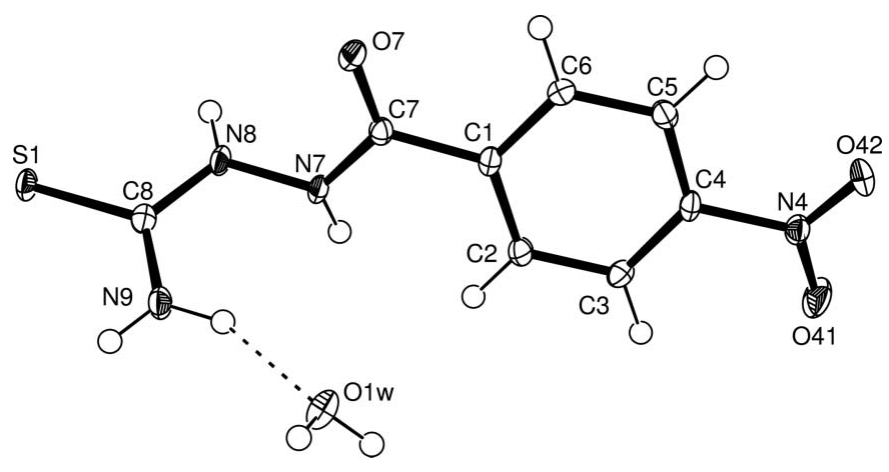

Figure 1

The molecular structure of the title compound, showing the atomlabelling scheme. Displacement ellipsoids are drawn at the $50 \%$ probability level. $\mathrm{H}$ atoms are shown as circles of arbitrary radius. The dashed line indicates a hydrogen bond.

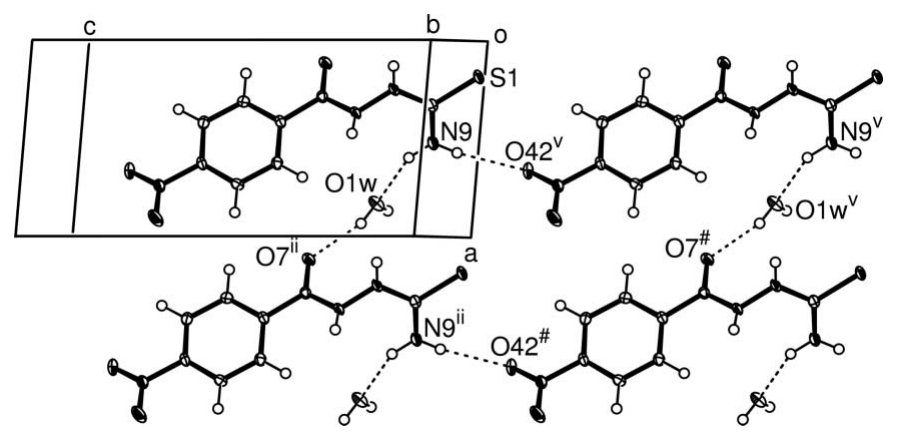

Figure 2

Part of the crystal structure of (I), showing the formation of a hydrogenbonded $R_{5}^{6}(34)$ ring which links with others to give sheets. Atoms marked with (ii), (v) or a hash (\#) are at the symmetry positions $(1+x, y, z),(x, y$, $-1+z)$ and $(1+x, y,-1+z)$, respectively. Dashed lines indicate hydrogen bonds.

[symmetry code: (i) $1-x, 1-y,-z$ ] to form an $R_{4}^{4}(12)$ ring. The other two are simpler motifs; N7-H7 . S $1^{\text {iii }}$ [symmetry code: (iii) $1-x, 2-y,-z$ ] giving an $R_{2}^{2}(10)$ ring and $\mathrm{N} 8-$ H8 $\cdots$ S1 $^{\text {iv }}$ [symmetry code: (iv) $-x, 2-y,-z$ ] forming an $R_{2}^{2}(8)$ motif. The former two dimers combine with the abovedescribed hydrogen bond to give a chain along [010] (Fig. 3). The sheet shown in Fig. 2 and the chain shown in Fig. 3 thus combine to give a three-dimensional hydrogen-bonded framework.

\section{Experimental}

A solution of potassium thiocyanate $(0.73 \mathrm{~g}, 12.5 \mathrm{mmol})$ and concentrated $\mathrm{HCl}(1.25 \mathrm{ml})$ was added to a stirred solution of 4nitrobenzoylhydrazide $(1.5 \mathrm{~g}, 8.3 \mathrm{mmol})$ (Hosamani \& Pattanashettar, 2004) in methanol $(21 \mathrm{ml})$. The mixture was evaporated to dryness on a steam bath, further methanol $(21 \mathrm{ml})$ was added and the mixture heated for $1 \mathrm{~h}$ on a steam bath. The resulting solid was successively washed with water and a small volume of ethanol, and recrystallized from acetone, yielding $2.1 \mathrm{~g}$ (70\%) of yellow 1-(4nitrobenzoyl)thiosemicarbazide (m.p. $489 \mathrm{~K}) .{ }^{1} \mathrm{H}$ NMR $(500 \mathrm{MHz}$, DMSO- $\left.d_{6}\right): \delta 10.71(s, 1 \mathrm{H}, \mathrm{CONHNH}), 9.44(s, 1 \mathrm{H}, \mathrm{CONH}), 8.33(d$, $2 \mathrm{H}, J=8.5 \mathrm{~Hz}, \mathrm{Ar}-\mathrm{H}), 8.13(d, 2 \mathrm{H}, J=8.5 \mathrm{~Hz}, \mathrm{Ar}-\mathrm{H}), 7.95(s, 1 \mathrm{H}$, $\left.\mathrm{CSNH}_{2}\right), 7.79\left(s, 1 \mathrm{H}, \mathrm{CSNH}_{2}\right)$.

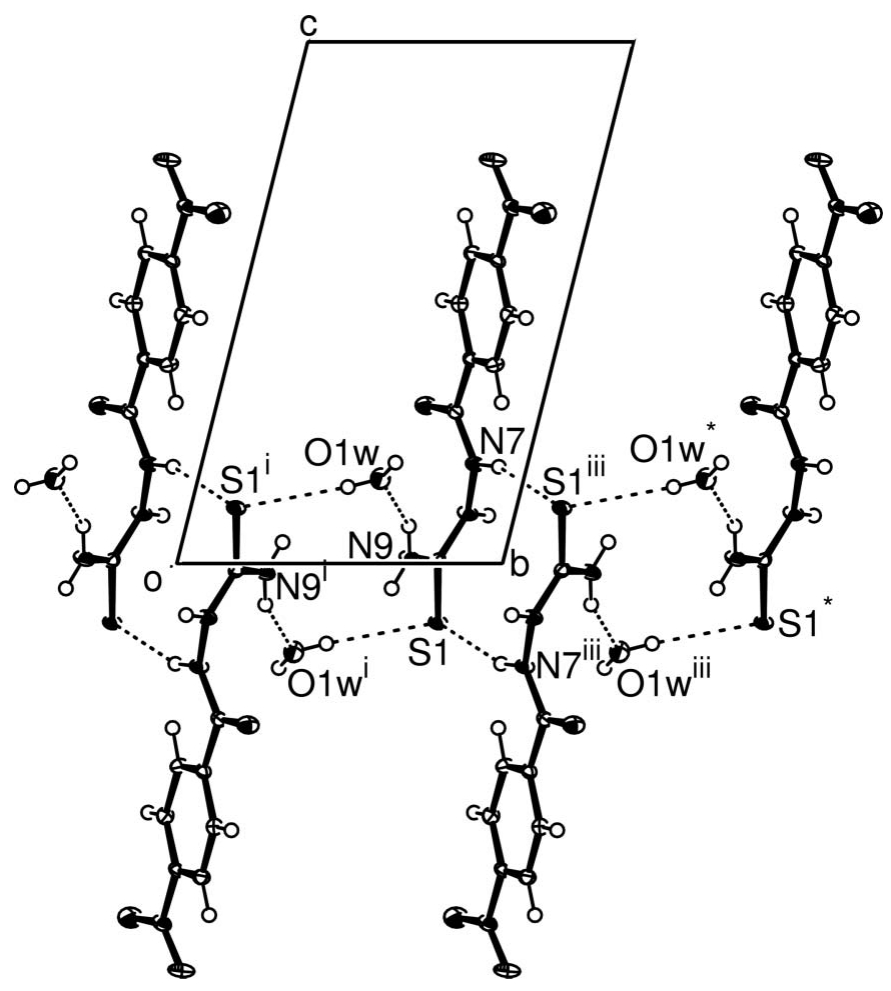

Figure 3

Part of the crystal structure of (I), showing the formation of hydrogenbonded dimers linked to form a chain. Atoms marked with (i), (iii) or an asterisk $(*)$ are at the symmetry positions $(1-x, 1-y,-z),(1-x, 2-y$, $-z)$ and $(x, 1+y, z)$ respectively. Dashed lines indicate hydrogen bonds.

Crystal data

$\mathrm{C}_{8} \mathrm{H}_{8} \mathrm{~N}_{4} \mathrm{O}_{3} \mathrm{~S} \cdot \mathrm{H}_{2} \mathrm{O}$

$M_{r}=258.26$

Triclinic, $P \overline{1}$

$a=6.0621(2) \AA$

$b=7.3991(3) \AA$

$c=12.2661(5) \AA$

$\alpha=75.9684(16)^{\circ}$

$\beta=85.112(2)^{\circ}$

$\gamma=88.903(2)^{\circ}$

$$
\begin{aligned}
& V=531.83(4) \AA^{3} \\
& Z=2 \\
& D_{x}=1.613 \mathrm{Mg} \mathrm{m}^{-3} \\
& \text { Mo } K \alpha \text { radiation }^{-1} \\
& \mu=0.32 \mathrm{~mm}^{-1} \\
& T=120(2) \mathrm{K} \\
& \text { Slab, pale yellow } \\
& 0.45 \times 0.45 \times 0.10 \mathrm{~mm}
\end{aligned}
$$

\section{Data collection}

Bruker-Nonius KappaCCD diffractometer

$\varphi$ and $\omega$ scans

Absorption correction: multi-scan (SADABS; Sheldrick, 2003)

$T_{\min }=0.688, T_{\max }=0.928$

\section{Refinement}

Refinement on $F^{2}$

$R\left[F^{2}>2 \sigma\left(F^{2}\right)\right]=0.031$

$w R\left(F^{2}\right)=0.091$

$S=1.12$

2425 reflections

172 parameters

$\mathrm{H}$ atoms treated by a mixture of independent and constrained refinement
8670 measured reflections 2425 independent reflections 2178 reflections with $I>2 \sigma(I)$ $R_{\text {int }}=0.028$ $\theta_{\max }=27.6^{\circ}$ 
Table 1

Hydrogen-bond geometry $\left(\AA,^{\circ}\right)$.

\begin{tabular}{lllll}
\hline$D-\mathrm{H} \cdots A$ & $D-\mathrm{H}$ & $\mathrm{H} \cdots A$ & $D \cdots A$ & $D-\mathrm{H} \cdots A$ \\
\hline $\mathrm{O} 1 W-\mathrm{H} 1 W A \cdots \mathrm{S} 1^{\mathrm{i}}$ & $0.79(2)$ & $2.61(2)$ & $3.3472(13)$ & $156.5(18)$ \\
$\mathrm{O} 1 W-\mathrm{H} 1 W A \cdots \mathrm{O} 7^{\mathrm{ii}}$ & $0.81(2)$ & $2.01(2)$ & $2.7944(15)$ & $162.7(19)$ \\
$\mathrm{N}-\mathrm{H} 7 \cdots \mathrm{S} 1^{\text {ii }}$ & $0.831(19)$ & $2.608(19)$ & $3.4096(13)$ & $162.4(16)$ \\
$\mathrm{N} 8-\mathrm{H} 8 \cdots \mathrm{S} 1^{\text {iv }}$ & $0.854(19)$ & $2.49(2)$ & $3.3382(13)$ & $172.0(16)$ \\
$\mathrm{N} 9-\mathrm{H} 9 A \cdots \mathrm{O} 42^{\mathrm{v}}$ & $0.84(2)$ & $2.26(2)$ & $3.0834(17)$ & $164.8(18)$ \\
$\mathrm{N} 9-\mathrm{H} 9 B \cdots \mathrm{O} 1 W$ & $0.89(2)$ & $1.94(2)$ & $2.7754(16)$ & $153.8(17)$ \\
\hline
\end{tabular}

Symmetry codes: (i) $-x+1,-y+1,-z$; (ii) $x+1, y, z$; (iii) $-x+1,-y+2,-z$; (iv) $-x,-y+2,-z ;$ (v) $x, y, z-1$.

All $\mathrm{H}$ atoms were located in difference maps; those in the aryl ring were then treated as riding atoms, with $\mathrm{C}-\mathrm{H}=0.95 \AA$ and $U_{\text {iso }}(\mathrm{H})=$ $1.2 U_{\text {eq }}(\mathrm{C})$. All other $\mathrm{H}$ atoms were refined freely.

Data collection: COLLECT (Hooft, 1998); cell refinement: DENZO (Otwinowski \& Minor, 1997) and COLLECT; data reduction: DENZO and COLLECT; program(s) used to solve structure: OSCAIL (McArdle, 2003) and SHELXS97 (Sheldrick, 1997); program(s) used to refine structure: OSCAIL and SHELXL97 (Sheldrick, 1997); molecular graphics: ORTEP-3 for Windows (Farrugia, 1997); software used to prepare material for publication: CIFTAB (Sheldrick, 1997) and PLATON (Spek, 2003).

We are indebted to the EPSRC for the use of both the Chemical Database Service at Daresbury, England, primarily for access to the Cambridge Structural Database (Fletcher et $a l ., 1996)$, and the X-ray service at the University of Southampton, England, for data collection.

\section{References}

Bernstein, J., Davis, R. E., Shimoni, L. \& Chang, N.-L. (1995). Angew. Chem. Int. Ed. Engl. 34, 1555-1573.

Bhat, A. K., Bhamaria, R. P., Bellare, R. A. \& Deliwala, C. V. (1967). Indian J. Chem. 5, 397-401.

Farrugia, L. J. (1997). J. Appl. Cryst. 30, 565.

Fletcher, D. A., McMeeking, R. F. \& Parkin, D. (1996). J. Chem. Inf. Comput. Sci. 36, 746-749.

Guersoy, A. \& Karali, N. (1995). Farmaco, 50, 857-866.

Hooft, R. W. W. (1998). COLLECT. Nonius BV, Delft, The Netherlands.

Hosamani, K. M. \& Pattanashettar, R. S. (2004). Ind. Eng. Chem. Res. 43, 4979-4999.

Kane, J. M., Staeger, M. A., Dalton, C. R., Miller, F. P., Dudley, M. W., Ogden, A. M., Kehne, J. H., Ketteler, H. J., Mccloskey, T. C., Senyah, Y., Chmielewski, P. A. \& Miller, J. A. (1994). J. Med. Chem. 37, 125-132.

McArdle, P. (2003). OSCAIL for Windows. Version 10. Crystallography Centre, Chemistry Department, National University of Ireland, Galway, Ireland.

Oruc, E. E., Rollas, S., Kandemirli, F., Shvets, N. \& Dimoglo, A. S. (2004). J. Med. Chem. 47, 6760-6767.

Otwinowski, Z. \& Minor, W. (1997). Methods in Enzymology, Vol. 276. Macromolecular Crystallography, Part A. edited by C. W. Carter Jr \& R. M. Sweet, pp. 307-326. New York: Academic Press.

Palaska, E., Sahin, G., Kelicen, P., Durlu, N. T. \& Altinok, G. (2002). Farmaco, 57, 101-107.

Plumitallo, A., Cardia, M. C., Distinto, S., DeLogu, A. \& Maccioni, E. (2004). Farmaco, 59, 945-952.

Sheldrick, G. M. (1997). SHELXS97, SHELXL97 and CIFTAB. University of Göttingen, Germany.

Sheldrick, G. M. (2003). SADABS. Version 2.10. University of Göttingen, Germany.

Shen, X., Wu, D., Huang, X., Liu, Q., Huang, Z. \& Kang, B. (1997). Polyhedron, 16, 1477-1482.

Singh, N. K. \& Singh, S. B. (2001). Indian J. Chem. Sect A, 40, 1070-1075.

Spek, A. L. (2003). J. Appl. Cryst. 36, 7-13.

Yale, H. L. \& Losee, K. (1966). J. Med. Chem. 9, 478-483. 


\section{supporting information}

Acta Cryst. (2006). E62, o2563-o2565 [https://doi.org/10.1107/S1600536806019301]

\section{1-(4-Nitrobenzoyl)thiosemicarbazide monohydrate: a three-dimensional hydrogen-bonded framework structure}

Núbia Boechat, Adriana Lages, W. Bruce Kover, Solange M. S. V. Wardell and Janet M. S. Skakle

1-(4-Nitrobenzoyl)thiosemicarbazide monohydrate

Crystal data

$\mathrm{C}_{8} \mathrm{H}_{8} \mathrm{~N}_{4} \mathrm{O}_{3} \mathrm{~S} \cdot \mathrm{H}_{2} \mathrm{O}$

$M_{r}=258.26$

Triclinic, $P \overline{1}$

Hall symbol: -P 1

$a=6.0621(2) \AA$

$b=7.3991(3) \AA$

$c=12.2661(5) \AA$

$\alpha=75.9684(16)^{\circ}$

$\beta=85.112(2)^{\circ}$

$\gamma=88.903(2)^{\circ}$

$V=531.83(4) \AA^{3}$

\section{Data collection}

Bruker-Nonius KappaCCD diffractometer

Radiation source: Bruker-Nonius KappaCCD

$10 \mathrm{~cm}$ confocal mirrors monochromator

Detector resolution: 9.091 pixels $\mathrm{mm}^{-1}$

$\varphi$ and $\omega$ scans

Absorption correction: multi-scan

(SADABS; Sheldrick, 2003)

$T_{\min }=0.688, T_{\max }=0.928$

\section{Refinement}

Refinement on $F^{2}$

Least-squares matrix: full

$R\left[F^{2}>2 \sigma\left(F^{2}\right)\right]=0.031$

$w R\left(F^{2}\right)=0.091$

$S=1.12$

2425 reflections

172 parameters

0 restraints

Primary atom site location: structure-invariant direct methods

$$
\begin{aligned}
& Z=2 \\
& F(000)=268 \\
& D_{\mathrm{x}}=1.613 \mathrm{Mg} \mathrm{m}^{-3}
\end{aligned}
$$

Mo $K \alpha$ radiation, $\lambda=0.71073 \AA$

Cell parameters from 2242 reflections

$\theta=2.9-27.5^{\circ}$

$\mu=0.32 \mathrm{~mm}^{-1}$

$T=120 \mathrm{~K}$

Slab, pale yellow

$0.45 \times 0.45 \times 0.10 \mathrm{~mm}$

8670 measured reflections

2425 independent reflections

2178 reflections with $I>2 \sigma(I)$

$R_{\text {int }}=0.028$

$\theta_{\max }=27.6^{\circ}, \theta_{\min }=3.4^{\circ}$

$h=-7 \rightarrow 7$

$k=-9 \rightarrow 9$

$l=-15 \rightarrow 15$

Secondary atom site location: difference Fourier map

Hydrogen site location: difference Fourier map

$\mathrm{H}$ atoms treated by a mixture of independent and constrained refinement

$w=1 /\left[\sigma^{2}\left(F_{\mathrm{o}}^{2}\right)+(0.0474 P)^{2}+0.2095 P\right]$

where $P=\left(F_{\mathrm{o}}{ }^{2}+2 F_{\mathrm{c}}{ }^{2}\right) / 3$

$(\Delta / \sigma)_{\max }<0.001$

$\Delta \rho_{\max }=0.37 \mathrm{e} \AA^{-3}$

$\Delta \rho_{\min }=-0.38$ e $\AA^{-3}$ 


\section{Special details}

Experimental. IR $v_{\max }\left(\mathrm{KBr}, \mathrm{cm}^{-1}\right): 3515,3429,3157,1683,1630,1605,1522,1348,1264,714 .{ }^{13} \mathrm{C} \mathrm{NMR}(125 \mathrm{MHz}$, DMSO- $\left.d_{6}\right): \delta 181.92,164.30,149.20,138.24,129.30,123.22$.

Geometry. All e.s.d.'s (except the e.s.d. in the dihedral angle between two 1.s. planes) are estimated using the full covariance matrix. The cell e.s.d.'s are taken into account individually in the estimation of e.s.d.'s in distances, angles and torsion angles; correlations between e.s.d.'s in cell parameters are only used when they are defined by crystal symmetry. An approximate (isotropic) treatment of cell e.s.d.'s is used for estimating e.s.d.'s involving 1.s. planes.

Refinement. Refinement of $F^{2}$ against ALL reflections. The weighted $R$-factor $w R$ and goodness of fit $S$ are based on $F^{2}$, conventional $R$-factors $R$ are based on $F$, with $F$ set to zero for negative $F^{2}$. The threshold expression of $F^{2}>\sigma\left(F^{2}\right)$ is used only for calculating $R$-factors (gt) etc. and is not relevant to the choice of reflections for refinement. $R$-factors based on $F^{2}$ are statistically about twice as large as those based on $F$, and $R$ - factors based on ALL data will be even larger.

Fractional atomic coordinates and isotropic or equivalent isotropic displacement parameters $\left(\AA^{2}\right)$

\begin{tabular}{lllll}
\hline & $x$ & $y$ & $z$ & $U_{\text {iso }}^{*} / U_{\text {eq }}$ \\
\hline C1 & $0.4146(2)$ & $0.75286(19)$ & $0.39533(11)$ & $0.0124(3)$ \\
C2 & $0.6269(2)$ & $0.82803(19)$ & $0.38368(11)$ & $0.0145(3)$ \\
H2 & 0.6979 & 0.8770 & 0.3106 & $0.019^{*}$ \\
C3 & $0.7348(2)$ & $0.83152(19)$ & $0.47840(12)$ & $0.0141(3)$ \\
H3 & 0.8784 & 0.8843 & 0.4714 & $0.018^{*}$ \\
C4 & $0.6275(2)$ & $0.75598(19)$ & $0.58338(11)$ & $0.0129(3)$ \\
C5 & $0.4188(2)$ & $0.67728(19)$ & $0.59826(12)$ & $0.0143(3)$ \\
H5 & 0.3507 & 0.6251 & 0.6716 & $0.019^{*}$ \\
C6 & $0.3118(2)$ & $0.67690(19)$ & $0.50267(12)$ & $0.0134(3)$ \\
H6 & 0.1677 & 0.6247 & 0.5103 & $0.017^{*}$ \\
N4 & $0.74170(19)$ & $0.75903(17)$ & $0.68434(10)$ & $0.0156(3)$ \\
O41 & $0.90680(19)$ & $0.85720(17)$ & $0.67255(9)$ & $0.0266(3)$ \\
O42 & $0.66489(18)$ & $0.66376(17)$ & $0.77561(9)$ & $0.0242(3)$ \\
C7 & $0.2872(2)$ & $0.74619(19)$ & $0.29655(11)$ & $0.0128(3)$ \\
O7 & $0.11625(16)$ & $0.65565(15)$ & $0.30868(9)$ & $0.0186(2)$ \\
N7 & $0.3713(2)$ & $0.84764(17)$ & $0.19467(10)$ & $0.0142(3)$ \\
H7 & $0.476(3)$ & $0.921(3)$ & $0.1890(15)$ & $0.018^{*}$ \\
N8 & $0.2502(2)$ & $0.86376(18)$ & $0.10162(10)$ & $0.0156(3)$ \\
H8 & $0.133(3)$ & $0.930(3)$ & $0.0979(15)$ & $0.020^{*}$ \\
C8 & $0.3325(2)$ & $0.80758(19)$ & $0.01032(11)$ & $0.0131(3)$ \\
N9 & $0.5224(2)$ & $0.71739(18)$ & $0.01309(11)$ & $0.0177(3)$ \\
H9A & $0.574(3)$ & $0.684(3)$ & $-0.0449(17)$ & $0.023^{*}$ \\
H9B & $0.598(3)$ & $0.689(3)$ & $0.0745(17)$ & $0.023^{*}$ \\
S1 & $0.18825(6)$ & $0.85785(5)$ & $-0.10755(3)$ & $0.01538(12)$ \\
O1W & $0.83048(19)$ & $0.56199(16)$ & $0.16378(10)$ & $0.0208(2)$ \\
H1WA & $0.932(3)$ & $0.595(3)$ & $0.1938(17)$ & $0.027^{*}$ \\
H1WA & $0.867(3)$ & $0.468(3)$ & $0.1476(17)$ & $0.027^{*}$ \\
& & & &
\end{tabular}

Atomic displacement parameters $\left(\AA^{2}\right)$

\begin{tabular}{lllllll}
\hline & $U^{11}$ & $U^{22}$ & $U^{33}$ & $U^{12}$ & $U^{13}$ & $U^{23}$ \\
\hline C1 & $0.0146(6)$ & $0.0122(6)$ & $0.0116(6)$ & $0.0024(5)$ & $-0.0043(5)$ & $-0.0042(5)$ \\
C2 & $0.0157(7)$ & $0.0159(7)$ & $0.0109(6)$ & $-0.0004(5)$ & $-0.0020(5)$ & $-0.0008(5)$ \\
C3 & $0.0125(6)$ & $0.0145(7)$ & $0.0157(7)$ & $-0.0013(5)$ & $-0.0033(5)$ & $-0.0032(5)$
\end{tabular}




\begin{tabular}{lllllll} 
C4 & $0.0162(7)$ & $0.0135(6)$ & $0.0107(6)$ & $0.0035(5)$ & $-0.0054(5)$ & $-0.0053(5)$ \\
C5 & $0.0160(7)$ & $0.0153(7)$ & $0.0115(6)$ & $0.0015(5)$ & $-0.0006(5)$ & $-0.0033(5)$ \\
C6 & $0.0120(6)$ & $0.0137(6)$ & $0.0146(7)$ & $0.0004(5)$ & $-0.0014(5)$ & $-0.0035(5)$ \\
N4 & $0.0160(6)$ & $0.0195(6)$ & $0.0134(6)$ & $0.0036(5)$ & $-0.0042(4)$ & $-0.0071(5)$ \\
O41 & $0.0258(6)$ & $0.0332(7)$ & $0.0223(6)$ & $-0.0090(5)$ & $-0.0111(4)$ & $-0.0060(5)$ \\
O42 & $0.0260(6)$ & $0.0371(7)$ & $0.0093(5)$ & $-0.0008(5)$ & $-0.0023(4)$ & $-0.0049(5)$ \\
C7 & $0.0137(6)$ & $0.0138(6)$ & $0.0119(6)$ & $0.0029(5)$ & $-0.0029(5)$ & $-0.0049(5)$ \\
O7 & $0.0153(5)$ & $0.0252(6)$ & $0.0158(5)$ & $-0.0047(4)$ & $-0.0040(4)$ & $-0.0046(4)$ \\
N7 & $0.0136(6)$ & $0.0192(6)$ & $0.0107(6)$ & $-0.0024(5)$ & $-0.0044(4)$ & $-0.0040(5)$ \\
N8 & $0.0151(6)$ & $0.0218(6)$ & $0.0111(6)$ & $0.0038(5)$ & $-0.0067(4)$ & $-0.0050(5)$ \\
C8 & $0.0157(7)$ & $0.0116(6)$ & $0.0112(6)$ & $-0.0032(5)$ & $-0.0021(5)$ & $-0.0009(5)$ \\
N9 & $0.0192(6)$ & $0.0238(7)$ & $0.0121(6)$ & $0.0066(5)$ & $-0.0059(5)$ & $-0.0071(5)$ \\
S1 & $0.0180(2)$ & $0.0187(2)$ & $0.01036(19)$ & $0.00160(13)$ & $-0.00624(13)$ & $-0.00371(13)$ \\
O1W & $0.0208(6)$ & $0.0193(6)$ & $0.0236(6)$ & $-0.0006(4)$ & $-0.0108(4)$ & $-0.0047(5)$ \\
& & & & & & \\
\hline
\end{tabular}

Geometric parameters $\left(\AA,^{\circ}\right)$

\begin{tabular}{|c|c|c|c|}
\hline $\mathrm{C} 1-\mathrm{C} 2$ & $1.3943(19)$ & $\mathrm{N} 4-\mathrm{O} 42$ & $1.2281(16)$ \\
\hline $\mathrm{C} 1-\mathrm{C} 6$ & $1.3973(19)$ & $\mathrm{C} 7-\mathrm{O} 7$ & $1.2234(17)$ \\
\hline $\mathrm{C} 1-\mathrm{C} 7$ & $1.5022(18)$ & $\mathrm{C} 7-\mathrm{N} 7$ & $1.3551(18)$ \\
\hline $\mathrm{C} 2-\mathrm{C} 3$ & $1.3867(19)$ & $\mathrm{N} 7-\mathrm{N} 8$ & $1.3890(16)$ \\
\hline $\mathrm{C} 2-\mathrm{H} 2$ & 0.9500 & $\mathrm{~N} 7-\mathrm{H} 7$ & $0.831(19)$ \\
\hline $\mathrm{C} 3-\mathrm{C} 4$ & $1.3840(19)$ & $\mathrm{N} 8-\mathrm{C} 8$ & $1.3411(18)$ \\
\hline $\mathrm{C} 3-\mathrm{H} 3$ & 0.9500 & N8- $\mathrm{H} 8$ & 0.854 (19) \\
\hline $\mathrm{C} 4-\mathrm{C} 5$ & $1.382(2)$ & $\mathrm{C} 8-\mathrm{N} 9$ & $1.3180(18)$ \\
\hline $\mathrm{C} 4-\mathrm{N} 4$ & $1.4742(17)$ & $\mathrm{C} 8-\mathrm{S} 1$ & $1.7135(14)$ \\
\hline $\mathrm{C} 5-\mathrm{C} 6$ & $1.3882(19)$ & N9-H9A & $0.84(2)$ \\
\hline $\mathrm{C} 5-\mathrm{H} 5$ & 0.9500 & N9-H9B & $0.89(2)$ \\
\hline C6- 6 6 & 0.9500 & $\mathrm{O} 1 \mathrm{~W}-\mathrm{H} 1 \mathrm{WA}$ & $0.81(2)$ \\
\hline $\mathrm{N} 4-\mathrm{O} 41$ & $1.2257(16)$ & $\mathrm{O} 1 \mathrm{~W}-\mathrm{H} 1 \mathrm{WA}$ & $0.79(2)$ \\
\hline $\mathrm{C} 2-\mathrm{C} 1-\mathrm{C} 6$ & $120.01(12)$ & $\mathrm{O} 41-\mathrm{N} 4-\mathrm{C} 4$ & $118.21(11)$ \\
\hline $\mathrm{C} 2-\mathrm{C} 1-\mathrm{C} 7$ & $123.07(12)$ & $\mathrm{O} 42-\mathrm{N} 4-\mathrm{C} 4$ & $118.00(12)$ \\
\hline $\mathrm{C} 6-\mathrm{C} 1-\mathrm{C} 7$ & $116.92(12)$ & $\mathrm{O} 7-\mathrm{C} 7-\mathrm{N} 7$ & $122.55(12)$ \\
\hline $\mathrm{C} 3-\mathrm{C} 2-\mathrm{C} 1$ & $120.21(12)$ & $\mathrm{O} 7-\mathrm{C} 7-\mathrm{C} 1$ & $121.35(12)$ \\
\hline $\mathrm{C} 3-\mathrm{C} 2-\mathrm{H} 2$ & 119.9 & $\mathrm{~N} 7-\mathrm{C} 7-\mathrm{C} 1$ & $116.10(12)$ \\
\hline $\mathrm{C} 1-\mathrm{C} 2-\mathrm{H} 2$ & 119.9 & $\mathrm{C} 7-\mathrm{N} 7-\mathrm{N} 8$ & $119.14(12)$ \\
\hline $\mathrm{C} 4-\mathrm{C} 3-\mathrm{C} 2$ & $118.22(12)$ & $\mathrm{C} 7-\mathrm{N} 7-\mathrm{H} 7$ & $121.3(12)$ \\
\hline $\mathrm{C} 4-\mathrm{C} 3-\mathrm{H} 3$ & 120.9 & $\mathrm{~N} 8-\mathrm{N} 7-\mathrm{H} 7$ & $117.8(13)$ \\
\hline $\mathrm{C} 2-\mathrm{C} 3-\mathrm{H} 3$ & 120.9 & $\mathrm{C} 8-\mathrm{N} 8-\mathrm{N} 7$ & $121.51(12)$ \\
\hline $\mathrm{C} 5-\mathrm{C} 4-\mathrm{C} 3$ & $123.23(13)$ & $\mathrm{C} 8-\mathrm{N} 8-\mathrm{H} 8$ & $119.3(12)$ \\
\hline $\mathrm{C} 5-\mathrm{C} 4-\mathrm{N} 4$ & $118.35(12)$ & N7-N8-H8 & $118.1(12)$ \\
\hline $\mathrm{C} 3-\mathrm{C} 4-\mathrm{N} 4$ & $118.42(12)$ & $\mathrm{N} 9-\mathrm{C} 8-\mathrm{N} 8$ & $119.39(13)$ \\
\hline $\mathrm{C} 4-\mathrm{C} 5-\mathrm{C} 6$ & $117.89(13)$ & $\mathrm{N} 9-\mathrm{C} 8-\mathrm{S} 1$ & $121.85(11)$ \\
\hline $\mathrm{C} 4-\mathrm{C} 5-\mathrm{H} 5$ & 121.1 & $\mathrm{~N} 8-\mathrm{C} 8-\mathrm{S} 1$ & $118.75(10)$ \\
\hline $\mathrm{C} 6-\mathrm{C} 5-\mathrm{H} 5$ & 121.1 & $\mathrm{C} 8-\mathrm{N} 9-\mathrm{H} 9 \mathrm{~A}$ & $118.7(13)$ \\
\hline $\mathrm{C} 5-\mathrm{C} 6-\mathrm{C} 1$ & $120.44(13)$ & $\mathrm{C} 8-\mathrm{N} 9-\mathrm{H} 9 \mathrm{~B}$ & $122.6(12)$ \\
\hline $\mathrm{C} 5-\mathrm{C} 6-\mathrm{H} 6$ & 119.8 & $\mathrm{H} 9 \mathrm{~A}-\mathrm{N} 9-\mathrm{H} 9 \mathrm{~B}$ & $118.7(17)$ \\
\hline
\end{tabular}




$\begin{array}{llll}\mathrm{C} 1-\mathrm{C} 6-\mathrm{H} 6 & 119.8 & \mathrm{H} 1 \mathrm{WA}-\mathrm{O} 1 \mathrm{~W}-\mathrm{H} 1 \mathrm{WA} & 107(2) \\ \mathrm{O} 41-\mathrm{N} 4-\mathrm{O} 42 & 123.78(12) & & \\ \mathrm{C} 6-\mathrm{C} 1-\mathrm{C} 2-\mathrm{C} 3 & -1.5(2) & \mathrm{C} 5-\mathrm{C} 4-\mathrm{N} 4-\mathrm{O} 42 & 12.54(18) \\ \mathrm{C} 7-\mathrm{C} 1-\mathrm{C} 2-\mathrm{C} 3 & 179.39(12) & \mathrm{C} 3-\mathrm{C} 4-\mathrm{N} 4-\mathrm{O} 42 & -167.28(13) \\ \mathrm{C} 1-\mathrm{C} 2-\mathrm{C} 3-\mathrm{C} 4 & 1.1(2) & \mathrm{C} 2-\mathrm{C} 1-\mathrm{C} 7-\mathrm{O} 7 & 169.10(13) \\ \mathrm{C} 2-\mathrm{C} 3-\mathrm{C} 4-\mathrm{C} 5 & 0.1(2) & \mathrm{C} 6-\mathrm{C} 1-\mathrm{C} 7-\mathrm{O} 7 & -10.07(19) \\ \mathrm{C} 2-\mathrm{C} 3-\mathrm{C} 4-\mathrm{N} 4 & 179.88(12) & \mathrm{C} 2-\mathrm{C} 1-\mathrm{C} 7-\mathrm{N} 7 & -11.73(19) \\ \mathrm{C} 3-\mathrm{C} 4-\mathrm{C} 5-\mathrm{C} 6 & -0.9(2) & \mathrm{C} 6-\mathrm{C} 1-\mathrm{C} 7-\mathrm{N} 7 & 5.9(2) \\ \mathrm{N} 4-\mathrm{C} 4-\mathrm{C} 5-\mathrm{C} 6 & 179.33(11) & \mathrm{O} 7-\mathrm{C} 7-\mathrm{N} 7-\mathrm{N} 8 & -173.27(11) \\ \mathrm{C} 4-\mathrm{C} 5-\mathrm{C} 6-\mathrm{C} 1 & 0.5(2) & \mathrm{C} 1-\mathrm{C} 7-\mathrm{N} 7-\mathrm{N} 8 & -121.29(15) \\ \mathrm{C} 2-\mathrm{C} 1-\mathrm{C} 6-\mathrm{C} 5 & 0.6(2) & \mathrm{C} 7-\mathrm{N} 7-\mathrm{N} 8-\mathrm{C} 8 & 7.2(2) \\ \mathrm{C} 7-\mathrm{C} 1-\mathrm{C} 6-\mathrm{C} 5 & 179.85(12) & \mathrm{N} 7-\mathrm{N} 8-\mathrm{C} 8-\mathrm{N} 9 & -172.06(10) \\ \mathrm{C} 5-\mathrm{C} 4-\mathrm{N} 4-\mathrm{O} 41 & -167.02(13) & \mathrm{N} 7-\mathrm{N} 8-\mathrm{C} 8-\mathrm{S} 1 & \\ \mathrm{C} 3-\mathrm{C} 4-\mathrm{N} 4-\mathrm{O} 41 & 13.16(19) & & \end{array}$

Hydrogen-bond geometry $\left(A,{ }^{\circ}\right)$

\begin{tabular}{lllll}
\hline$D-\mathrm{H} \cdots A$ & $D-\mathrm{H}$ & $\mathrm{H} \cdots A$ & $D \cdots A$ & $D-\mathrm{H}^{\cdots} A$ \\
\hline $\mathrm{O} 1 W-\mathrm{H} 1 W A \cdots \mathrm{S} 1^{\mathrm{i}}$ & $0.79(2)$ & $2.61(2)$ & $3.3472(13)$ & $156.5(18)$ \\
$\mathrm{O} 1 W-\mathrm{H} 1 W A \cdots 7^{\mathrm{ii}}$ & $0.81(2)$ & $2.01(2)$ & $2.7944(15)$ & $162.7(19)$ \\
$\mathrm{N} 7-\mathrm{H} 7 \cdots \mathrm{S} 1^{\mathrm{iii}}$ & $0.831(19)$ & $2.608(19)$ & $3.4096(13)$ & $162.4(16)$ \\
$\mathrm{N} 8-\mathrm{H} 8 \cdots \mathrm{S}^{\mathrm{iv}}$ & $0.854(19)$ & $2.49(2)$ & $3.3382(13)$ & $172.0(16)$ \\
$\mathrm{N} 9-\mathrm{H} 9 A \cdots \mathrm{O} 42^{\mathrm{v}}$ & $0.84(2)$ & $2.26(2)$ & $3.0834(17)$ & $164.8(18)$ \\
$\mathrm{N} 9-\mathrm{H} 9 B \cdots \mathrm{O} 1 W$ & $0.89(2)$ & $1.94(2)$ & $2.7754(16)$ & $153.8(17)$ \\
\hline
\end{tabular}

Symmetry codes: (i) $-x+1,-y+1,-z$; (ii) $x+1, y, z$; (iii) $-x+1,-y+2,-z$; (iv) $-x,-y+2,-z$; (v) $x, y, z-1$. 